\title{
Triplet Stabilization for the Enhanced Drug Photorelease from Sunscreen-Based Photocages
}

Received 00th January 20xx, Accepted 00th January 20xx

DOI: $10.1039 / x 0 x \times 00000 x$

\author{
Mauricio Lineros-Rosa, ${ }^{a}$ M. Consuelo Cuquerella, ${ }^{a}$ Antonio Francés-Monerris, ${ }^{b, c}$ Antonio Monari, ${ }^{* b}$ \\ Miguel A. Miranda*a and Virginie Lhiaubet-Vallet*a
}

\begin{abstract}
Recently, sunscreen-based drug photocages have been introduced to provide UV protection to photoactive drugs, thus increasing their photosafety. Here, combined experimental and theoretical studies performed on a photocage based on the commercial UVA filter avobenzone $(\mathrm{AB})$ and on the photosensitizing non-steroidal anti-inflammatory drug ketoprofen (KP) are presented unveiling the photophysical processes responsible for the light-triggered release. Particular attention is paid to solvent stabilization of the drug and UV filter excited states, respectively, which leads to a switching between the triplet excited state energies of the $A B$ and KP units. Most notably, we show that the stabilization of the $A B$ triplet excited state in ethanol solution is the key requirement for an efficient photouncaging. By contrast, in apolar solvents, in particular hexane, $\mathrm{KP}$ has the lowest triplet excited state, hence acting as an energy acceptor quenching the $A B$ triplet manifold, thus inhibiting the desired photoreaction.
\end{abstract}

\section{Introduction}

Significant advances have been made since the early reports on photoremovable protecting groups (also known as photolabile protecting groups, PPG), ${ }^{1-3}$ which are now essential in a multitude of research fields as diverse as organic synthesis, catalysis, therapeutic application, imaging, sensing, modulation of protein-DNA binding, etc. ${ }^{4-14}$ The main advantage of PPG is the use of light as an external and non-invasive stimulus, which results in minimal secondary processes and offers a fully complementary alternative to conventional chemical and biological tools. In addition, the photorelease can be performed with a high spatiotemporal control and without additional chemicals, thus holding promise for the development of a wide range of applications in organic chemistry, biochemistry, biology, polymer science, lithography, toiletry, etc. ${ }^{4-14}$

Phenacyl derivatives are among the most used PPG as they provide an extensive toolkit for protection of chemical functions such as carboxylic acids, alcohols, thiols, phosphates, sulfonates, amines, etc. ${ }^{4,10,15,16}$ They operate through different mechanisms including inter- or intramolecular $\mathrm{H}$-abstraction, electron transfer from suitable donors or photo-Favorskii rearrangement. However, in most of these processes, triplet excited states have been established as the key intermediates. ${ }^{4}$ Thus, an efficient intersystem crossing (ISC) is often a prerequisite for high photoprotecting efficacy. Recently,

a. Instituto Universitario Mixto de Tecnologia Química UPV-CSIC, Universitat Politècnica de València, Consejo Superior de Investigaciones Científicas, Avda de los Naranjos s/n, 46022 Valencia, Spain.

b. Université de Lorraine and CNRS, LPCT UMR 7019, F-54000 Nancy

c. Departament de Química Física Universitat de València 46100 Burjassot, Spain

† Footnotes relating to the title and/or authors should appear here.

Electronic Supplementary Information (ESI) available: [details of any supplementary information available should be included here]. See DOI: 10.1039/x0xx00000x

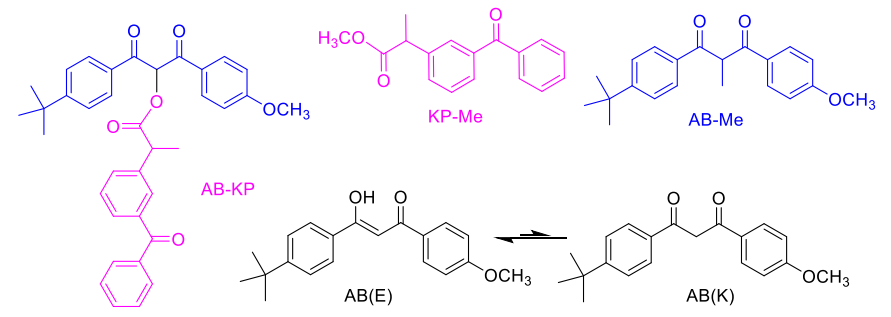

Chart 1. Structure of the studied compounds

the dibenzoylmethane derivative avobenzone ( $A B, 4$-tert-butyl-4methoxydibenzoylmethane, Chart 1 ), has been proposed as a valuable phenacyl-like PPG for carboxylic acid photorelease. ${ }^{17,18}$ Interestingly, this compound not only behaves as an ordinary PPG, i.e. being released upon irradiation, but it also presents an added value since it acts as a well-established UVA filter. Indeed, $A B$ is already largely used to achieve photoprotection in commercial sunscreens and cosmetics formulations. This combined action has given rise to the new concept of synergic prodrug/profilter, which results in a perfect combination to provide the phototriggered delivery of a photosensitive drug together with its UVA protective filter. Indeed, this concept has been successfully proven with ketoprofen and diclofenac, two highly photosensitive non-steroidal anti-inflammatory drugs (NSAIDs) ${ }^{19,20}$ used as topical pain relievers. ${ }^{17,18}$ Photochemical studies have demonstrated the controlled and simultaneous photorelease of the drug (KP) and the filter (AB). More specifically, the latter is generated as its diketo form, $A B(K)$, but it rapidly undergoes a keto-enol isomerization that generates the thermodynamically favored enol tautomer, $A B(E)$, as outlined in Chart 1. The $A B(E)$ form is responsible for the large 
UVA absorption, due to the formation of intramolecular hydrogen bond that enforces the planarity of the molecular skeleton as compared to $A B(K)$, thus increasing the $\pi$ conjugation, red-shifting the absorption spectrum and providing photoprotection. ${ }^{21,22}$ Indeed, thanks to the filtering action of $A B(E)$, the NSAID photoreactivity is inhibited and, consequently, the risk of adverse skin reactions, such as phototoxicity and photoallergy, is minimized.

In the AB-KP systems, the photorelease process has been proposed to take place from the excited triplet state of the $A B$ moiety in its diketonic form, ${ }^{3} A B(K)^{*}$, following a reaction mechanism similar to the one previously established for phenacyl derivatives. ${ }^{4}$ Hence, the efficient population of ${ }^{3} \mathrm{AB}(\mathrm{K}) *$ appears essential to reach the optimal conditions for the photorelease. Since AB-KP can be partitioned into two basic constituent fragments, namely $A B-M e$ and KP-Me (Chart 1 ), the energetic order of the triplet states centered on one or another unit can be crucial to dictate the global photodissociation efficiency. More precisely, under the photoreaction conditions, the triplet excited state centered on KP-Me ( $\left.{ }^{3} \mathrm{KP}-\mathrm{Me}^{*}\right)$ should be located at a higher energy than the one localized over AB-Me ( $\left.{ }^{3} \mathrm{AB}-\mathrm{Me}{ }^{*}\right)$; otherwise, a triplet-triplet energy transfer toward KP-Me would occur, deactivating the photoreactive ${ }^{3} \mathrm{AB}-\mathrm{Me}{ }^{*}$ - while populating the non-reactive ${ }^{3} \mathrm{KP}-\mathrm{Me}{ }^{*}$-centered triplet. It is worthy to mention that both fragments are based on aromatic ketones, whose excited state energies are in general sensitive to solvent polarity, especially when their electronic configuration is of $n \pi^{*}$ nature. ${ }^{23}$

With this background, in the present work, thorough spectroscopic and molecular modeling studies are employed to assess the importance of the solvent in the relative location of ${ }^{3} \mathrm{AB}(\mathrm{K})^{*}$ versus ${ }^{3} \mathrm{KP} *$. Specifically, the photophysics of the prodrug/profilter dyad AB$K P$ has been assessed in hexane and ethanol and compared with the properties of its basic components, namely AB-Me and KP-Me. Our results reveal that the change in the environment polarity is associated with a noticeable change of the triplet state energetic order, hence leading to totally different photophysical outcomes that correlate with a remarkable modification in the efficiency of the photouncaging process.

\section{Experimental}

Chemicals. AB-KP, AB-Me and KP-Me were prepared as previously described in the literature. ${ }^{17,21,24}$

UV-Vis absorption. UV absorption spectra were registered on a Cary 50 spectrophotometer (Varian) using a quartz cuvette of $1 \mathrm{~cm}$ optical path and $3 \mathrm{~mL}$ capacity.

Steady-state photolysis. Simulated sunlight (SSL) irradiation, experiments were carried out with a Thermo Oriel Newport (A91192A) solar simulator equipped with a $1000 \mathrm{~W}$ Xe arc. Its output was adequately filtered to produce a spectrum approximating natural sunlight (1.5 G air mass filter). The spectral output was measured as ca. $1000 \mathrm{~mW} / \mathrm{cm}^{2}$.

Monitoring of the UV-Vis spectral changes as a function of SSL irradiation time was performed for deaerated solutions of $A B-K P$ at ca. $7 \times 10^{-5} \mathrm{M}$ in hexane or ethanol.

Laser flash photolysis (LFP). Experiments were run with a pulsed Nd:YAG (L52137 V LOTIS TII) laser system instrument setting $355 \mathrm{~nm}$ as excitation wavelength. The pulse duration was of ca. $10 \mathrm{~ns}$ and the energy was adjusted at $20 \mathrm{~mJ}$ pulse $\mathrm{s}^{-1}$. The apparatus consisted of the pulsed laser, the Xe lamp, a 77250 Oriel monochromator, and a photomultiplier. The output signal from a Tektronix oscilloscope was transferred to a personal computer. The transient spectra were recorded at room temperature employing quartz cells of $1 \mathrm{~cm}$ optical path length. Experiments were performed for ethanol and hexane solutions of AB-KP ( $3.6 \times 10^{-4}$ and $3 \times 10^{-4} \mathrm{M}$, respectively) under $\mathrm{N}_{2}$ atmosphere. Fresh solutions were used for each pulse to avoid degradation of $A B-K P$ during the experiment.

In ethanol, the photorelease rate constant $(k)$ was determined by using the equation: $1 / \tau_{0}=1 / \tau+k$, where $\tau_{0}$ is lifetime of unreactive ${ }^{3} \mathrm{AB} *$ and $\tau$ is the lifetime of ${ }^{3} \mathrm{AB}-\mathrm{KP} *$.

Femtosecond transient absorption spectroscopy. The transient absorption spectra were recorded using a typical pump-probe system. The femtosecond pulses were generated with a compact regenerative amplifier that produces pulses centered at $800 \mathrm{~nm}$ ( $100 \mathrm{fs}, 1 \mathrm{~mJ} /$ pulse). The output of the laser was split into two parts to generate the pump and the probe beams. Thus, tunable femtosecond pump pulses were obtained by directing the $800 \mathrm{~nm}$ light into an optical parametric amplifier. In the present case, the pump was set at $340 \mathrm{~nm}$ and passed through a chopper prior to focus onto a rotating cell containing the solutions under study. The white light used as probe was produced after part of the $800 \mathrm{~nm}$ light from the amplifier travelled through a computer controlled 8 ns variable optical delay line and impinge on a $\mathrm{CaF}_{2}$ rotating crystal. This white light is in turn, split in two identical portions to generate reference and probe beams that then are focused on the rotating cell (optical pathway of ca. $0.8 \mathrm{~mm}$ ) containing the sample.

The pump and the probe are made to coincide to interrogate the sample. A computer-controlled imaging spectrometer is placed after this path to measure the probe and the reference pulses and obtain the transient absorption decays/spectra. Sample were prepared with an absorbance of ca. 1 at the pump wavelength, using an optical pathway of $1 \mathrm{~mm}$.

Molecular modeling. All optimizations were performed at the Density Functional Theory (DFT) level using $\omega B$ B $7-X^{25}$ as exchange correlation functional and the $6-31 \mathrm{G}(\mathrm{d})$ basis set.

Solvation (ethanol and hexane) has been taken into account implicitly via the continuum polarizable method (PCM) 26 as implemented in the Gaussian 0927 software using the default parameters.

Excited-state energies and equilibrium geometries have been obtained using the Time-Dependent DFT (TD-DFT) method making use of the Tamm-Dancoff Approximation (TDA) ${ }^{28}$ in order to avoid triplet instability problems. ${ }^{29}$ The singlet and triplet electronic densities have been analyzed in terms of Natural Transition Orbitals (NTOs) ${ }^{30}$ making use of the NANCY_EX code. ${ }^{31,32}$

\section{Results and Discussion}

\section{Steady-state analysis of the photorelease process}

In a first stage, the influence of the solvent on the photorelease was evaluated using ethanol and hexane, two solvents with different polarities (dielectric constant, $\varepsilon$, of ca. 24.55 and 1.89, respectively) ${ }^{33}$ and $\mathrm{H}$-donor capabilities. Thus, $\mathrm{N}_{2}$ deaerated solutions of AB-KP (7 $\mathrm{x}$ $10^{-5} \mathrm{M}$ ) were irradiated with simulated sunlight (SSL), and the course 

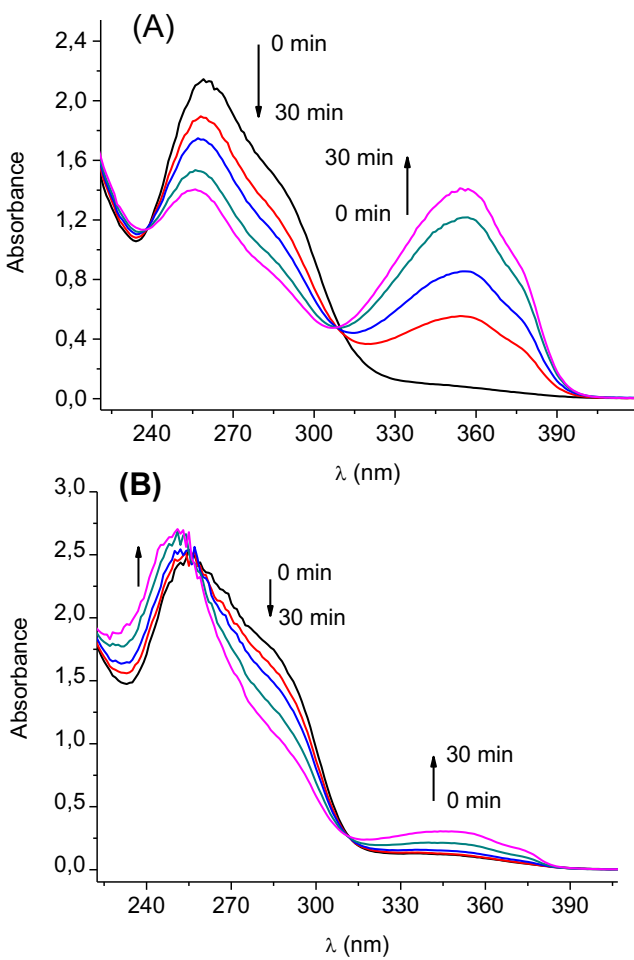

Figure 1. UV absorption changes for $A B-K P\left(7 \times 10^{-5} \mathrm{M}\right)$ in deaerated ethanol (A) or hexane (B) under SSL irradiation (from 0 to $30 \mathrm{~min}$ )

of the reaction was followed by UV-Vis absorption spectrophotometry monitoring the photorelease of $A B$ through the appearance of its characteristic $355 \mathrm{~nm}$ absorption band. ${ }^{17}$ Irradiation performed in ethanol solution resulted in an evident increase of the intensity of the $A B(E)$ signature band with irradiation time (Figure 1A). ${ }^{17}$ By contrast, the irradiation of the hexane solutions showed a markedly slower photorelease. Indeed, even after 30 min of irradiation the $A B(E)$ UVA band is barely noticeable and more specifically seven-fold lower than for ethanol solutions (Figure 1B). Further experiments, performed in acetonitrile (Figure S1) showed the inefficiency of the photorelease process when a polar solvent (dielectric constant, $\varepsilon$, of ca. 35.94$)^{33}$ with poor $\mathrm{H}$-donor capability is used. Thus, results in hexane could, in a first approximation, be explained by the lack of $\mathrm{H}$-donor capability of hexane, which is a crucial step to assure the release mechanism. However, an alternative explanation can be considered taking into account that the photorelease is initiated by the population of the phenacyl-like triplet excited state ${ }^{3} \mathrm{AB}(\mathrm{K}) *{ }^{*}{ }^{17}$ In this context, aromatic ketones are well-known for the marked solvent-dependence of the energetic levels of their excited states, especially for the $n \pi^{*}$ ones. ${ }^{23}$ Thus, we performed spectroscopic analysis and theoretical calculations to get deeper insight into the lowest singlet and triplet excited state landscape when ethanol and hexane are used as solvents.
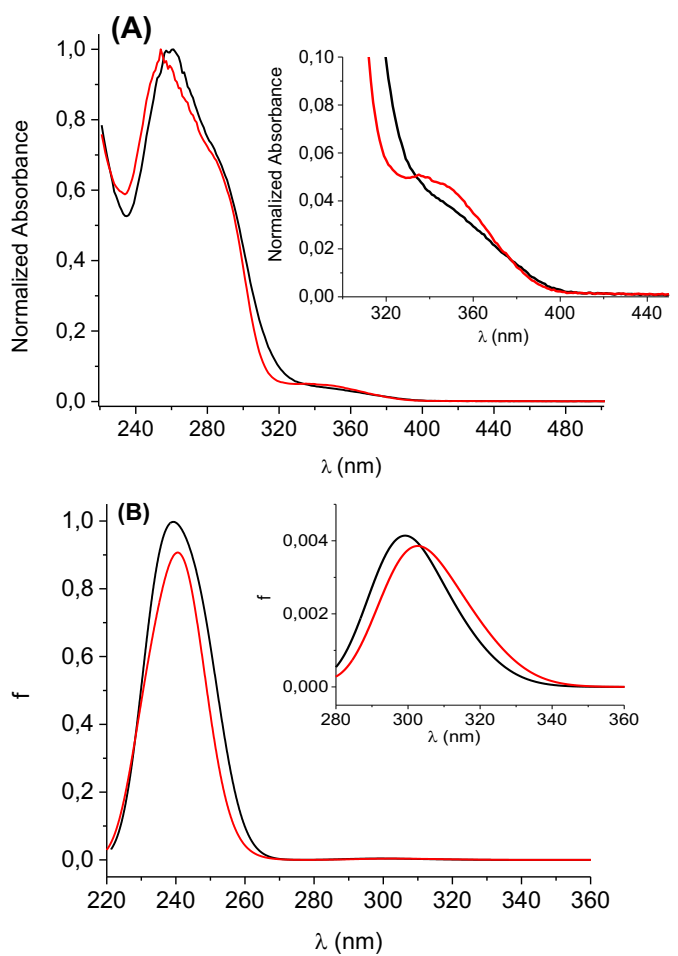

Figure 2. (A) Normalized UV-Vis absorption spectra of AB-KP in hexane (red) and ethanol (black). Inset: zoom of the absorption in the 300-450 nm range. (B) Calculated absorption spectrum (10 excited States) of the dyad in ethanol and hexane. Inset: absorption in the UVA region.

\section{Spectroscopic and computational study of the excitation}

Solvatochromism, due to the singlet excited states ordering, was studied for AB-KP and its basic chromophores AB-Me and KP-Me using UV-Vis absorption spectroscopy. In the case of the dyad, the main absorption band, located in the UVC, experiences only a weak bathochromic shift (from 254 to $260 \mathrm{~nm}$ ) when changing the solvent from hexane to ethanol, which is consistent with a transition of $\pi \pi^{*}$ nature (Figure 2). By contrast, a shoulder, observed in the UVA at $\sim 355 \mathrm{~nm}$, is shifted to the blue and becomes clearly more resolved when hexane is used as solvent (Figure $2 A$, inset), confirming its $n \pi^{*}$ character. Concerning the isolated chromophores of the dyad, ABMe and KP-Me (Figure S2), they also present both UVC and UVA absorption bands of $\pi \pi^{*}$ and $n \pi^{*}$ nature, ${ }^{21,34}$ respectively, which exhibit the typical shift, associated with their respective electronic configurations, as a function of solvent polarity.

The nature of the transitions was also confirmed by the calculation of the absorption spectra (Figure 2B) obtained as vertical transitions from an ensemble of geometries of the Franck-Condon region. The bands reproduce the hypsochromic shift of the lowest-energy transitions with the increase of the solvent polarity. A more detailed analysis of the electronic density reorganization confirms the presence of an almost dark $\left(\mathrm{S}_{1}, \mathrm{n} \pi^{*}\right)$ and a much brighter $\left(\pi \pi^{*}\right)$ 


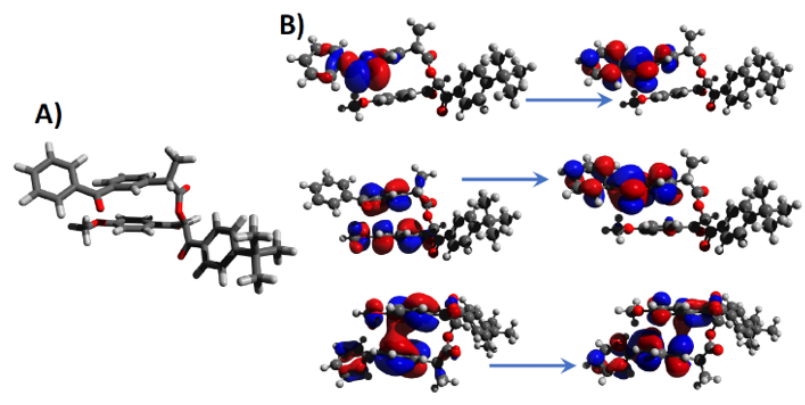

Figure 3. (A) DFT equilibrium geometry for the ground state of the AB-KP dyad. Note the $\pi$-stacking between the AB and KP units. (B) Hole (left) and particle (right) NTOs for three lowest-lying singlet excited states.

absorption. As can be seen from the natural transition orbitals (NTOs) reported in Figure 3, the $\pi$-stacking between the $A B$ and KP chromophores also induces an excitonic coupling leading to the delocalization of the hole and electron densities over the two moieties.

\section{Transient intermediates in the nanosecond-microsecond timescale}

As mentioned above, triplet excited states are key species in the photochemistry of phenacyl-based PPG. Therefore, to evaluate the population and reactivity of ${ }^{3} \mathrm{AB}-\mathrm{KP} *,{ }^{3} \mathrm{AB}-\mathrm{Me}^{*}$ and ${ }^{3} \mathrm{KP}-\mathrm{Me}{ }^{*}$, laser flash photolysis experiments were performed by means of a nanosecond pulsed laser (Nd:YAG) using $355 \mathrm{~nm}$ as excitation wavelength. Transient absorption spectra obtained for deaerated solutions of KP-Me in both solvents are in agreement with those described in the literature for ketoprofen. ${ }^{34,35}$ In ethanol, the typical benzophenone-like behavior was observed with the generation of a very short-lived triplet excited state centered at $520 \mathrm{~nm}$ ( $\tau$ of ca. 0.05 $\mu \mathrm{s})$ that gives rise to the formation of the ketyl radical peaking at 550 $\mathrm{nm}$ (Figure S3). While the same species were observed in hexane, the triplet decay was slower with a characteristic time $\tau$ of ca. $0.4 \mu \mathrm{s}$. Deaerated ethanol or hexane solutions of $A B-M e$ showed only one transient absorption peaking at ca. $400 \mathrm{~nm}$ and with lifetime of ca. 1.3 and $0.4 \mu \mathrm{s}$, respectively, which was assigned to the triplet excited state of $A B-M e$ by comparison with the literature data. ${ }^{21}$. No signal corresponding to ketyl radical formation was detected under the experimental conditions. (see note ${ }^{\ddagger}$ )

Interestingly, in the case of $A B-K P$, a marked difference of the spectra was observed depending on the solvent (Figure 4). In ethanol, a transient absorption peaking at $400 \mathrm{~nm}$ was detected (Figure $4 \mathrm{~A}$ ) and assigned, by comparison with $\mathrm{AB}-\mathrm{Me}$, to the triplet-triplet transition of the avobenzone-like moiety in its diketo form ${ }^{3} \mathrm{AB}(\mathrm{K})^{*}$. A negative signal was also observed, it might be explained by ground state bleaching coupled to the effect of $355 \mathrm{~nm}$ laser excitation. The $400 \mathrm{~nm}$ band decays in the sub- $\mu$ s range with a lifetime $\tau$ of ca. $0.2 \mu \mathrm{s}$. At longer times (ie. $0.9 \mu$ s after the laser pulse), a weak signal, similar to the one of ketoprofen ketyl radical, was observed at ca. $550 \mathrm{~nm}$. Interestingly, the $400 \mathrm{~nm}$ transient absorption was the only
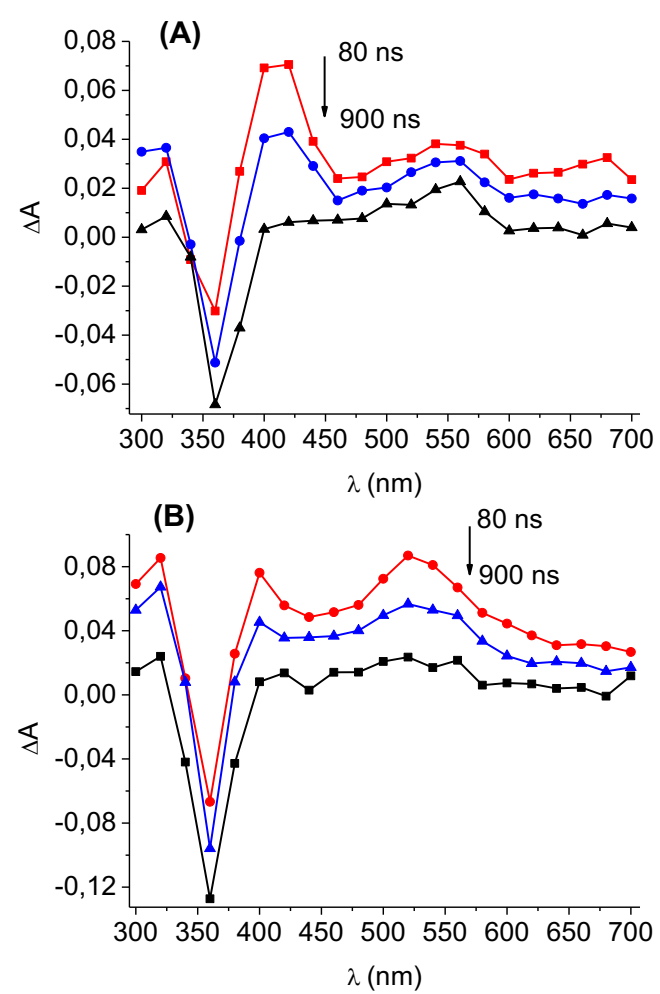

Figure 4. Transient absorption spectra of $A B-K P$ in ethanol (A) or in hexane (B) under $\mathrm{N}_{2}$, at different times after the $355 \mathrm{~nm}$ laser pulse.
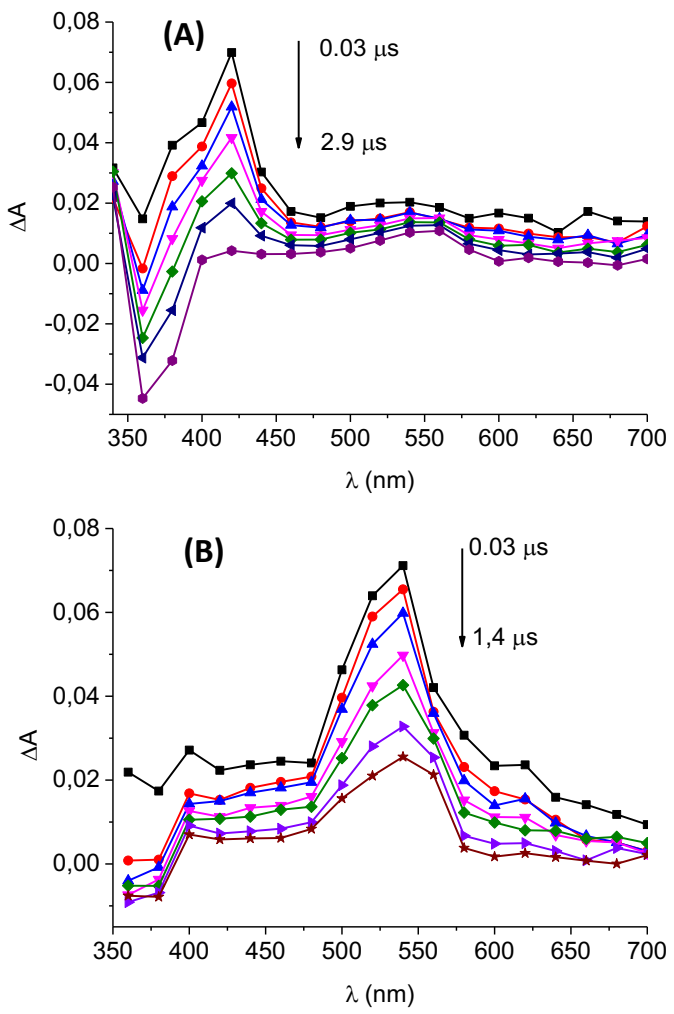

Figure 5. Transient absorption spectra of a degassed 1:1 mixture of AB-Me:KP-Me in ethanol (A) or in hexane (B) at different time after the $355 \mathrm{~nm}$ laser pulse. 
signal obtained in deaerated acetonitrile (Figure S1). By contrast, in hexane (Figure $4 B$ ), the $A B(K)$ triplet band at $400 \mathrm{~nm}$ was not present to a significant extent, while a transient absorption at ca. $520 \mathrm{~nm}$ similar to the well-known triplet absorption of KP was detected decaying without (apparently) giving rise to any ketyl radical formation.

In order to investigate whether this intriguing behavior was only observable in the covalently bound AB-KP dyad, the photophysical study was also extended to mixtures of 1:1 (3 $\mathrm{mM}: 3 \mathrm{mM}$ ) ratio of AB-Me and KP-Me in either deaerated EtOH or hexane (Figure 5). At $355 \mathrm{~nm}$, most of the incoming light is absorbed by AB-Me ( $64 \%$ in EtOH or $72 \%$ in hexane).

Interestingly, the transient absorption spectra observed in both solvents are quite similar to those obtained for the AB-KP dyad. In $\mathrm{EtOH},{ }^{3} \mathrm{AB}-\mathrm{Me}{ }^{*}$ and some ketyl radical (that could arise from direct irradiation of the KP moiety), were detected (Figure 5A). In the 1:1 mixture the lifetime of the species responsible for the $400 \mathrm{~nm}$ was $0.8 \mu \mathrm{s}$, which corresponds to a bimolecular quenching rate constant of ca. $10^{8} \mathrm{M}^{-1} \mathrm{~s}^{-1}$. The hexane spectra, as in the case of the dyad, only exhibited the triplet-triplet transition of ${ }^{3} \mathrm{KP}-\mathrm{Me}{ }^{*}$ with $\tau$ of ca. $0.4 \mu \mathrm{s}$, evidencing a very efficient quenching of ${ }^{3} \mathrm{AB}-\mathrm{Me}{ }^{*}$ by $\mathrm{KP}-\mathrm{Me}$ through a triplettriplet energy transfer process (Figure $5 B$ ).

\section{Analysis of the sub-nanosecond processes}

In order to analyze the sub-nanosecond processes and to shed light on the population of ${ }^{3} \mathrm{KP} *$ in hexane, ultrafast transient absorption spectroscopy was employed. At this timescale, KP-Me alone in ethanol (Figure S4, upper panel) or in hexane (Figure S4, bottom panel) exhibited the characteristic singlet-singlet transition at 580 $\mathrm{nm}$, which gave rise to the $525 \mathrm{~nm}$ triplet-triplet absorption band through an efficient intersystem crossing in less than 20 ps. ${ }^{17}$

As shown in Fig. 6A, the signals derived from KP singlet-singlet or triplet-triplet transient absorption were hardly detected for the dyad $\mathrm{AB}-\mathrm{KP}$ in ethanol or hexane within the first $20 \mathrm{ps}$. At long delay times (up to $3 \mathrm{~ns}$ ), no new band appeared in the $500-620 \mathrm{~nm}$ region when the spectrum was obtained in ethanol (Figure 6B).

Notably, when hexane was used as solvent the same behavior was detected for AB-KP up to 50 ps (Figure 7A); however, after this delay time, a signal centered at $525 \mathrm{~nm}$ emerges (Fig. 7B). It is noteworthy that, in the dyad, the characteristic singlet-singlet transient absorption of KP was not detected, and the KP triplet state absorption band increased with a time constant higher than in the case of KP alone (Figure S4, bottom). This supports formation of ${ }^{3} \mathrm{KP} *$ in hexane through a triplet-triplet energy transfer from ${ }^{3} \mathrm{AB}(\mathrm{K})^{*}$, rather than by intersystem crossing from the corresponding excited singlet state, a process that was not detected in ethanol. A possible explanation for this solvent-related difference would be an inversion of the relative excited state energy levels of $A B(K)$ and $K P$, which in the case of hexane could favor energy transfer from $A B$ to $K P$.

\section{Computational study of triplet excited states}

The above results are further supported by the exploration of the triplet potential energy surfaces of the AB-KP dyad at a timedependent density functional theory (TD-DFT) level. As shown in Figure 8 and Table S1, the two lowest-lying triplet states are centered
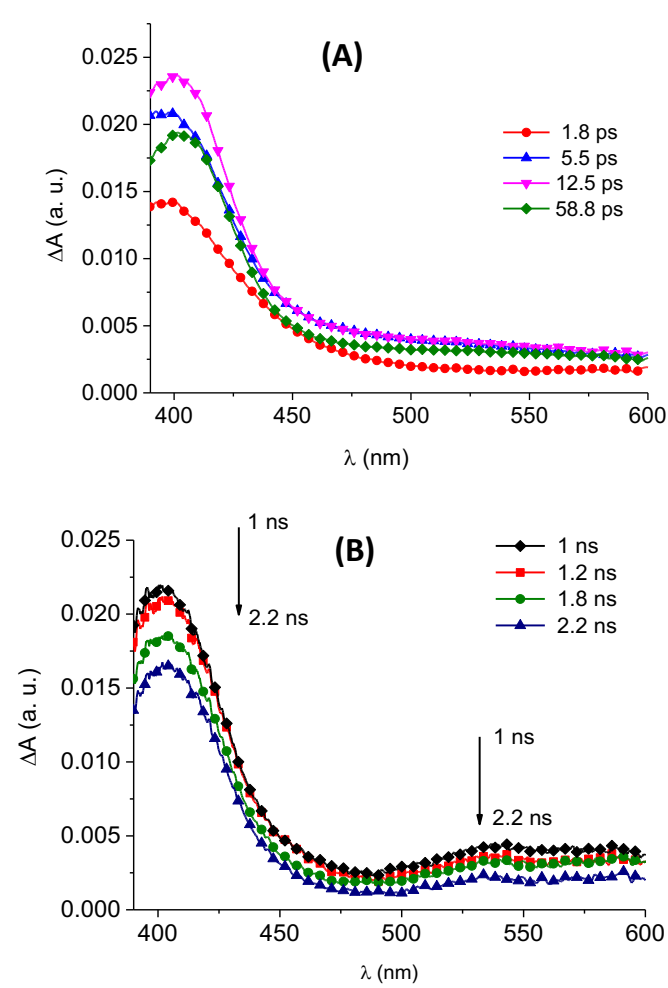

Figure 6. Transient absorption spectra of $A B-K P$ in ethanol (A) from $1.8 \mathrm{ps}$ to $58.8 \mathrm{ps}$ and (B) from $1 \mathrm{~ns}$ to $2.2 \mathrm{~ns}$ after delay time
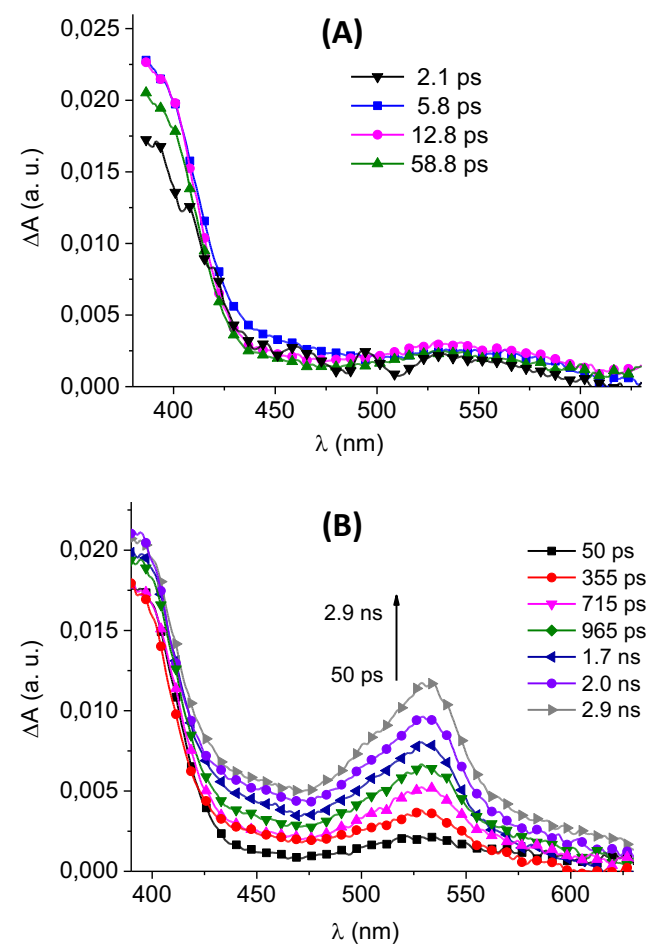

Figure 7. Transient absorption spectra of $A B-K P$ in hexane $(A)$ from 0.3 ps to $58.8 \mathrm{ps}$ and (B) from 50 ps to $2.0 \mathrm{~ns}$ after pump excitation 
over the KP and the $A B$ subsystems, as revealed by the analysis of their spin densities and the NTOs defining the lone-pair electrons (Figure $8 \mathrm{C}$ ). At the Franck-Condon geometry, irrespectively of the solvent, ${ }^{3} \mathrm{KP} *$ is always lower in energy than ${ }^{3} \mathrm{AB}$. However, the energy gap between them is closer in ethanol due to the increase of solvent polarity.

Upon triplet geometry optimization, while the state of interest is strongly stabilized, a noticeable increase in energy is observed for the other one (Figure 8A,B and Table S1). In hexane, when considering the corresponding adiabatic energy difference, ${ }^{3} \mathrm{KP} *$ is the lowest state, being $0.06 \mathrm{eV}$ lower than ${ }^{3} \mathrm{AB}^{*}$. Conversely, the energetic ordering of the states is inverted in the case of ethanol, in which ${ }^{3} \mathrm{AB}$ at its equilibrium geometry is now $0.02 \mathrm{eV}$ more stable than ${ }^{3} \mathrm{KP} *$. Even though the energy differences are small, the nature of the states (Figure $8 \mathrm{C}$ ) correlate well with the different photophysics experienced as a function of the solvent described in detail by the experimental measurements. Indeed, after the initial intersystem crossing, leading most probably to the population of ${ }^{3} \mathrm{KP} *$, the diabatic nature of the triplet state is conserved in hexane, while in ethanol the most stable triplet localized over ${ }^{3} A B^{*}$ supports a formal triplet-triplet energy transfer towards this molecular moiety. The observed small energy difference raises the question of the behaviour of the dyad in intermediate polarity solvents, or in inhomogeneous media. Even if the precise characterization of such complex systems goes clearly beyond the scope of the present contribution, we may speculate that a competition between the pathways leading to the population of ${ }^{3} \mathrm{KP} *$ or ${ }^{3} A B^{*}$ could be envisaged that in turn could affect the photouncaging quantum yield. Even if the energetic barrier related to the triplet transfer has not been explicitly modelled here, the relatively small energy difference, and the rather moderate geometric reorganization, should point towards a relatively facile and a fast process, coherently with the spectroscopic measurements.

\section{Mechanistic proposal}

Altogether these results show the importance of the triplet excited state location for the photouncaging process. Indeed, ${ }^{3} \mathrm{AB}-\mathrm{Me}{ }^{*}$ and ${ }^{3} \mathrm{KP}-\mathrm{Me}{ }^{*}$ are both $\mathrm{n} \pi *$ triplets, which energies are influenced by the solvent polarity.

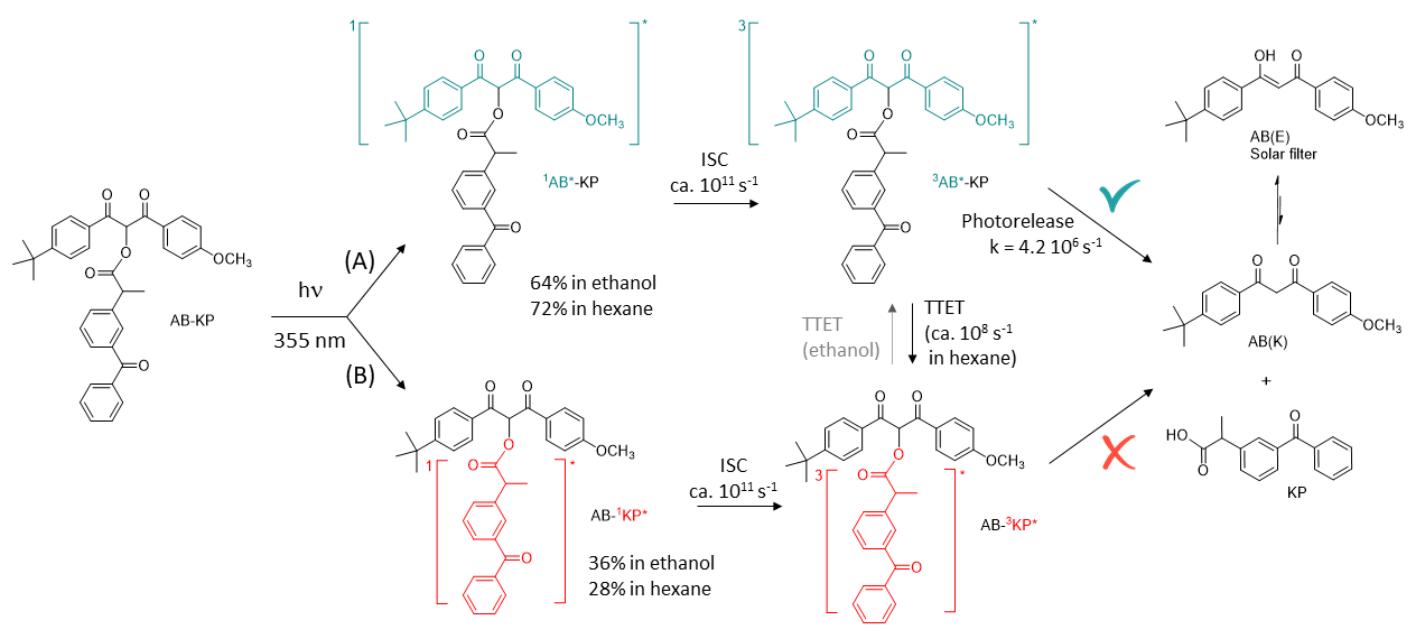

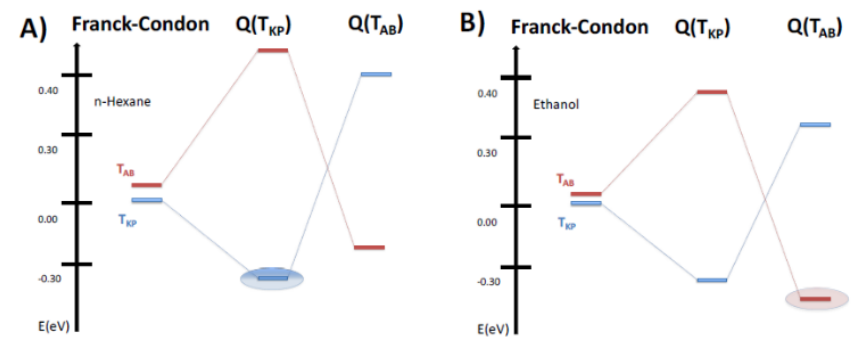

c)

$T_{\text {KP }}$

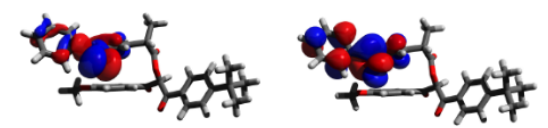

$T_{A B}$

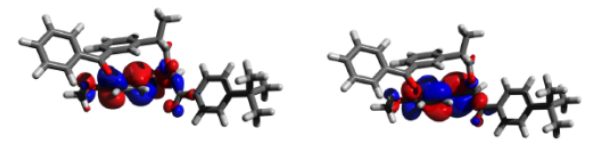

Figure 8. Energy diagram of the lowest triplet states of the dyad in hexane (A) and ethanol (B) and NTOs describing the lone pair electrons of the triplet state centered on the ketoprofen $\left({ }^{3} \mathrm{KP} *\right)$ and avobenzone $\left({ }^{3} A B^{*}\right)$ moiety, respectively $(C)$. All energies are relative to the triplet state localized over $\mathrm{KP}\left({ }^{3} \mathrm{KP} *\right)$ at the Franck-Condon region.

This could provoke an inversion of their relative triplets leading, in few $\mu s-n s$, to an excitation located on one or another of the fragments.

In this context, understanding of the deactivation pathways of $A B-K P$ is key to optimize the photorelease, the population of the reactive ${ }^{3} A B(K)^{*}$ being essential to assure the final step after the ultrafast non-radiative processes.As shown in Scheme 1, after excitation, two different routes are possible depending on the excited chromophore. In the case of a $355 \mathrm{~nm}$ irradiation, most of the light is absorbed by $A B(64 \%$ and $72 \%$ at $355 \mathrm{~nm}$ in ethanol and hexane, respectively). Pathway $A$ leads to formation of a singlet excited state localized on the $A B(K)$ moiety, followed by efficient population of ${ }^{3} A B(K)^{*}$, which finally gives rise to the photouncaging process.

Scheme 1. Photodeactivation processes of AB-KP. 
If the light is absorbed by the KP moiety, a similar route (pathway B) takes place giving rise to KP singlet and triplet excited states. However, no photorelease is expected from this pathway.

In hexane, experimental and theoretical data have clearly demonstrated that the lowest triplet is the KP one. Excitation of $A B$ results in the formation, after ISC, of its triplet excited state ${ }^{3} \mathrm{AB}(\mathrm{K})^{*}$. However, an efficient triplet-triplet energy transfer (TTET) from ${ }^{3} \mathrm{AB}(\mathrm{K})$ * to the KP moiety deactivates the reactive phenacyl-like triplet and decreases the proportion of the photorelease. Indeed, this latter process, with a rate constant $\mathrm{k}$ of ca. $4.210^{6} \mathrm{~s}^{-1}$, competes with TTET ( $\mathrm{k}$ of ca. $10^{8} \mathrm{~s}^{-1}$ ), and thus cannot represent more than $4 \%$ of the deactivation paths. The TTET process is clearly evidenced by the ultrafast transient absorption experiments, which show a delayed formation of ${ }^{3} \mathrm{KP} *$ beginning $50 \mathrm{ps}$ after the laser pulse. This has been unequivocally associated with TTET. Indeed, the lack of a ${ }^{1} \mathrm{KP} *$ signal at a short and long timescale is in complete agreement with this mechanism, ruling out not only the formation of ${ }^{3} \mathrm{KP} *$ by direct excitation, but also as a consequence of a singletsinglet energy transfer between ${ }^{1} \mathrm{AB}(\mathrm{K})^{*}$ and $\mathrm{KP}$, followed by ISC. The TTET is moreover in accordance with the computational results that establish a ${ }^{3} \mathrm{KP} *$ as the lowest state as compared to the ${ }^{3} \mathrm{AB}(\mathrm{K}) *$ excitation. Once, populated, ${ }^{3} \mathrm{KP} *$ should deactivate through nonradiative processes or give rise to the typical BP-like photochemistry (ie. mainly photoreduction).

By contrast, in ethanol, the excitation remains localized on the $A B(K)$ moiety as demonstrated by the spectroscopic studies with the only detection of the ${ }^{3} \mathrm{AB}(\mathrm{K})^{*}$ signal at $400 \mathrm{~nm}$, and the occurrence of efficient photorelease. This trend is again confirmed by TD-DFT calculations, which demonstrated that the ${ }^{3} \mathrm{AB}(\mathrm{K})$ excitation is lower in energy with respect to the ${ }^{3} \mathrm{KP} *$ state.

Further evidence for the importance of polarity and $\mathrm{H}$-donor capability of the solvent is given by experiments performed in acetonitrile (polar, poor $\mathrm{H}$-donor), where no photorelease takes place, or in propylene glycol (polar, good H-donor), where photorelease is observed, even under aerobic conditions. ${ }^{17}$

\section{Conclusions}

Mechanistic studies of a sunscreen-based drug photocage containing the UVA-filter avobenzone and the photosensitizing drug ketoprofen are reported. Combined spectroscopic analysis and molecular modeling constitute an unambiguous proof for the solvent-dependent energies of the drug and filter triplet excited states. Stabilization of avobenzone triplet manifold in polar solvents is essential for an efficient photorelease. By contrast, in nonpolar solvents the triplet localization is reversed,

since the triplet is in this case centered over ketoprofen. This opens the door to a deactivation of avobenzone excited state through triplet-triplet energy transfer toward ketoprofen, inactivating the photouncaging process. The obtained results are of high value for the design and the optimization of the photorelease conditions of new sunscreen-based drug photocages.

\section{Conflicts of interest}

There are no conflicts to declare.

\section{Acknowledgements}

Support from the Université de Lorraine, CNRS, regional (Prometeo/2017/075) and Spanish Government (PGC2018096684-B-I00, CTQ2017-87054-C2-2-P) is kindly acknowledged. A. F.-M. is grateful to Generalitat Valenciana and the European Social Fund (postdoctoral contract APOSTD/2019/149 and project GV/2020/226) for financial support. M. L.-R. acknowledges the Universitat Politècnica de València for the FPI grant. All calculations have been performed on the local LPCT computer center and on the Explor regional center in the framework of the project "Dancing under the light".

\section{Notes and references}

₹ Laser flash photolysis experiments performed on AB-Pr (a propionate derivative of $A B$ ) have shown a triplet-triplet transient absorption at $400 \mathrm{~nm}$ (see Figure S7) similar to ${ }^{3} \mathrm{AB}-\mathrm{Me}^{*}$ signal. However, the presence of the ester functional group makes this compound photolabile. In this work, we have used AB-Me as a photostable model of the $A B(K)$ moiety.

1
J. A. Barltrop and P. Schofield, J. Chem. Soc., 1965, 47584765.

J. A. Barltrop, P. J. Plant and P. Schofield, Chem. Commun., 1966, 822-823.

J. C. Sheehan and K. Umezawa, J. Org. Chem., 1973, 38, 3771-3774.

P. Klán, T. Šolomek, C. G. Bochet, A. Blanc, R. Givens, M. Rubina, V. Popik, A. Kostikov and J. Wirz, Chem. Rev., 2013, 113, 119-191.

J. M. Silva, E. Silva and R. L. Reis, J. Control. Release, 2019 , 298, 154-176.

A. Herrmann, Angew. Chem. Int. Ed., 2007, 46, 5836-5863. Y. Wu, Z. Yang and Y. Lu, Curr. Opin. Chem. Biol., 2020, 57, 95-104.

R. S. Stoll and S. Hecht, Angew. Chem. Int. Ed., 2010, 49, 
5054-5075.

9 M. Hocek, Acc. Chem. Res., 2019, 52, 1730-1737.

10 E. Speckmeier and K. Zeitler, ACS Catal., 2017, 7, 68216826.

K. A. Korzycka, P. M. Bennett, E. J. Cueto-Diaz, G. Wicks, M. Drobizhev, M. Blanchard-Desce, A. Rebane and H. L. Anderson, Chem. Sci., 2015, 6, 2419-2426. M. J. Hansen, W. A. Velema, M. M. Lerch, W. Szymanski and B. L. Feringa, Chem. Soc. Rev., 2015, 44, 3358-3377. M. Klausen, V. Dubois, J.-B. Verlhac and M. BlanchardDesce, Chempluschem, 2019, 84, 589-598.

C. Brieke, F. Rohrbach, A. Gottschalk, G. Mayer and A. Heckel, Angew. Chem. Int. Ed., 2012, 51, 8446-8476. Y. Q. Yang, Z. Lu and X. Xu, Asian J. Org. Chem., 2019, 8, 2192-2195. E. Speckmeier, M. Klimkait and K. Zeitler, J. Org. Chem., 2018, 83, 3738-3745.

I. Aparici-Espert, M. C. Cuquerella, C. Paris, V. LhiaubetVallet and M. A. Miranda, Chem. Commun., 2016, 52, 14215-14218.

I. Aparici-Espert, M. A. Miranda and V. Lhiaubet-Vallet, Molecules, 2018, 23, 673.

V. Lhiaubet-Vallet and M. A. Miranda, in CRC Handbook of Organic Photochemistry and Photobiology., eds. F. Ghetti, A. G. Griesbeck and M. Oelgemöller, CRC Press, 2012, pp. 1541-1555.

E. Bignon, M. Marazzi, V. Besancenot, H. Gattuso, G. Drouot, C. Morell, L. A. Eriksson, S. Grandemange, E. Dumont and A. Monari, Sci. Rep., 2017, 7, 8885. C. Paris, V. Lhiaubet-Vallet, O. Jiménez, C. Trullas and M. Á. Miranda, Photochem. Photobiol., 2009, 85, 178-184. V. Lhiaubet-Vallet, M. Marin, O. Jimenez, O. Gorchs, C. Trullas and M. A. Miranda, Photochem. Photobiol. Sci., 2010, 9, 552-558. N. J. Turro, V. Ramamurthy and Scaiano J. C., Principles of Molecular Photochemistry: An Introduction, University Science Books, 2009. C. D.-T. Nielsen, A. J. P. White, D. Sale, J. Bures and A. C. Spivey, J. Org. Chem., 2019, 84, 14965-14973. J. Da Chai and M. Head-Gordon, Phys. Chem. Chem. Phys., 2008, 10, 6615-6620. J. Tomasi, B. Mennucci and R. Cammi, Chem. Rev., 2005, 105, 2999-3093.

D. J. Frisch, M. J.; Trucks, G.W.; Schlegel, H. B.; Scuseria, G. E.; Robb, M. A.; Cheeseman, J. R.; Scalmani, G.; Barone, V.;Mennucci, B.; Petersson, G. A.; Nakatsuji, H.; Caricato, M.; Li, X.; Hratchian, H. P.; Izmaylov, A. F.; Bloino, J.; Zheng, G.; Sonnenber, Gaussian, Inc. Wallingford CT, 2009, 2-3. S. Hirata and M. Head-Gordon, Chem. Phys. Lett., 1999, 314, 291-299. M. J. G. Peach, M. J. Williamson and D. J. Tozer, J. Chem. Theory Comput., 2011, 7, 3578-3585. R. L. Martin, J. Chem. Phys., 2003, 118, 4775-4777.
M. Montalti, A. Credi, L. Prodi and M. Teresa Gandolfi, Handbook of Photochemistry, Third Edition, CRC Press, 2006.

34 V. Lhiaubet, F. Gutierrez, F. Penaud-Berruyer, E. Amouyal, J. P. Daudey, R. Poteau, N. Chouini-Lalanne and N. Paillous, New J. Chem., 2000, 24, 403-410. Biol., 1998, 43, 1-26.

T. Etienne, X. Assfeld and A. Monari, J. Chem. Theory Comput., 2014, 10, 3896-3905.

T. Etienne, X. Assfeld and A. Monari, J. Chem. Theory Comput., 2014, 10, 3906-3914. 